\title{
L'huître, un coquillage nomade sans tête ni jambe mais avec un pied
}

The oyster, a nomad shellfish with no head nor leg but a foot

\section{Pascale Legué et Jean Prou}

\section{(2) OpenEdition}

\section{Journals}

Édition électronique

URL : https://journals.openedition.org/tc/6717

DOI : $10.4000 /$ tc. 6717

ISSN : 1952-420X

Éditeur

Éditions de l'EHESS

\section{Édition imprimée}

Date de publication : 15 décembre 2012

Pagination : 284-305

ISBN : 978-2-7351-1534-1

ISSN : 0248-6016

\section{Référence électronique}

Pascale Legué et Jean Prou, «L'huître, un coquillage nomade sans tête ni jambe mais avec un pied », Techniques \& Culture [En ligne], 59 | 2012, mis en ligne le 15 décembre 2015, consulté le 29 septembre 2022. URL : http://journals.openedition.org/tc/6717; DOI : https://doi.org/10.4000/tc.6717 


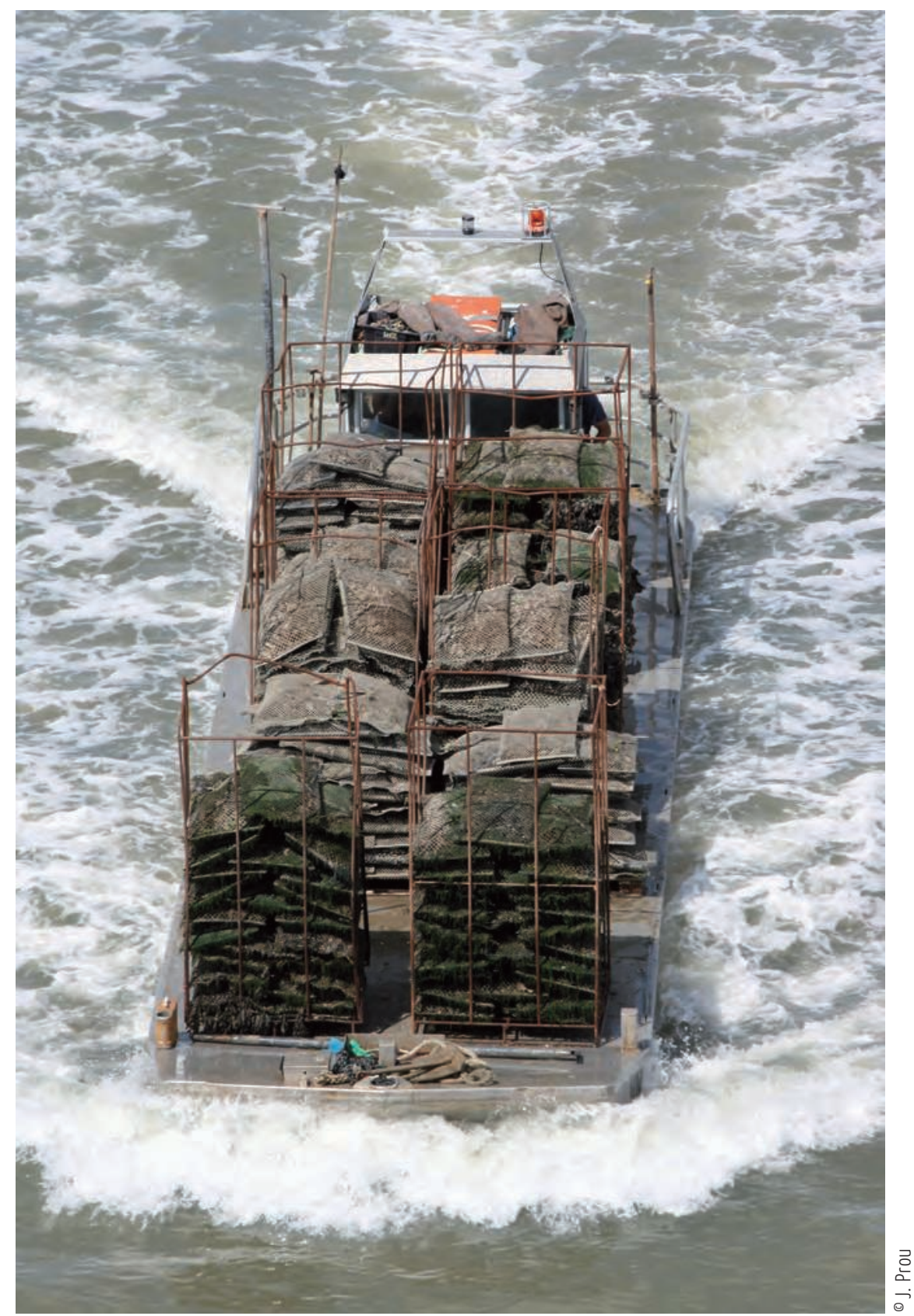




\section{L'HUITTRE, UN COQUILLAGE NOMADE SANS TÊTE NI JAMBE MAIS AVEC UN PIED}

Source d'inspiration pour les uns ou mets exquis et raffiné pour les autres, laissons aux poètes et aux gastronomes le soin d'apprécier ce coquillage. Pour le biologiste, la vie de l'huître est objet d'analyse. Pour l'ethnologue, bien que l'huître en soi ne soit pas sujet de recherche, elle en reste l'argument sociologique, ce coquillage étant constitutif d'un groupe social. L'éthologie de l'huître n'a de sens et n'est saisissable que réintégrée dans une pratique sociale dont elle livre la compréhension.

La destinée de l'huître - produit vivant - a une résonance capitale sur les mondes sociaux qui l'exploitent. La disparition d'une espèce et l'adoption d'une autre poussent à la constitution de nouveaux groupes professionnels, déplacent l'activité ostréicole vers de nouveaux espaces (estran*, établissement*, marais* ou autres bassins), entraînent des bouleversements techniques, culturels mais aussi scientifiques, majeurs.

Malgré les apparences, l'huître est un coquillage itinérant. Ses voyages dans les eaux françaises en témoignent. La larve de l'huître est d'abord une éphémère globe-trotteuse. Elle se laisse porter par les eaux et les courants vers son lieu de résidence où elle va s'ancrer à l'aide d'un pied qu'elle va perdre pour devenir un coquillage fixé. En effet, l'huître sauvage est par nature un coquillage sédentaire. Sans jambe ni tête, sa seule « pensée » est de vivre dans des lieux où elle trouve à se nourrir sans se mouvoir. Pourtant, la nature mais aussi l'homme vont l'inviter à parcourir les eaux, à traverser le monde, et cela depuis les Romains. L'homme va aussi, après domestication, lui faire vivre des moments de migration* et de transhumance* auxquels ce coquillage s'adapte d'ailleurs fort bien.

Pour la société française du XIxe au xxi siècle, l'huître a pour seules destinées, l'alimentaire et la marchandisation. Les appétences de l'homme (consommateur, scientifique,
L'huître, de la grosseur d'un galet moyen, est d'une apparence plus rugueuse, d'une couleur moins unie, brillamment blanchâtre. C'est un monde opiniâtrement clos. Pourtant on peut l'ouvrir: il faut alors la tenir au creux d'un torchon, se servir d'un couteau ébréché et peu franc, s’y reprendre à plusieurs fois. Les doigts curieux s'y coupent, s'y cassent les ongles: c'est un travail grossier. Les coups qu'on lui porte marquent son enveloppe de ronds blancs, d'une sorte de halo. À l'intérieur l'on trouve tout un monde, à boire et à manger: sous un firmament (à proprement parler) de nacre, les cieux d'en dessus s'affaissent sur les cieux d'en dessous, pour ne plus former qu'une mare, un sachet visqueux et verdâtre, qui flue et reflue à l'odeur et à la vue, frangé d'une dentelle noirâtre sur les bords. Parfois très rare une formule perle à leur gosier de nacre, d'où l'on trouve aussitôt à s'orner.

Francis Ponge, Le parti pris des choses, "L'huître » 


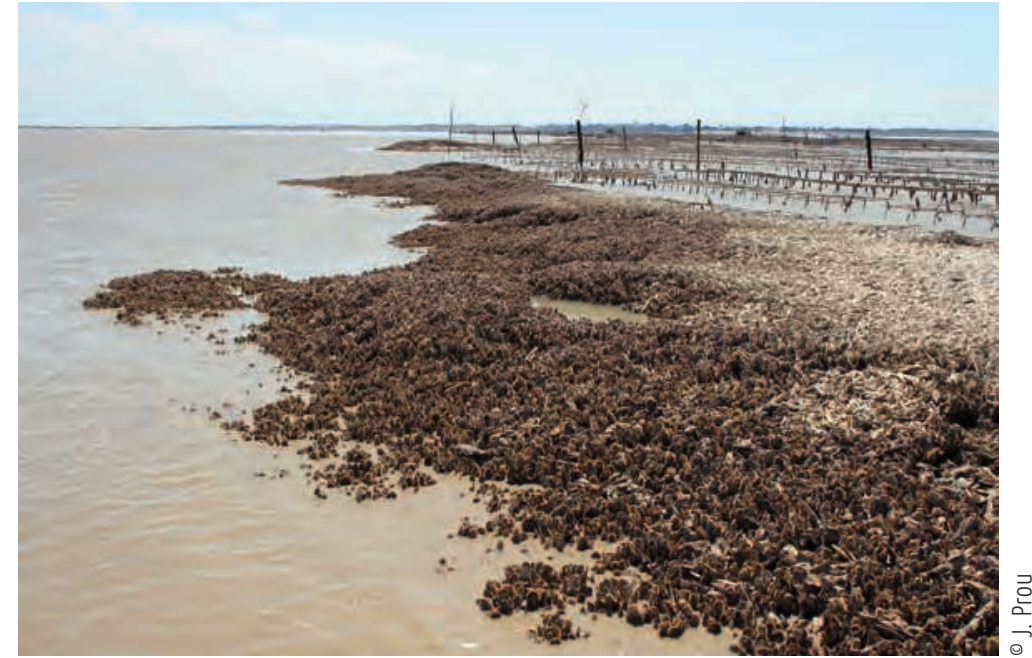

Avant la naissance de l'ostréiculture, les bancs d'huîtres ou huîtrières ont été pêchés et dragués au gré des besoins de l'homme pour son alimentation ou pour les nécessités du commerce. Ces bancs sont aussi le réservoir des huîtres-mères, géniteurs qui assurent la reproduction de l'espèce. producteur) sont de bonifier sa chair pour en optimiser la comestibilité.

Par ses désirs de transformer trop avant sa nature sauvage - sédentaire, sans tête, ni membres - pour en faire un produit commercial véhiculé sur tous les marchés, l'humain fragilise régulièrement l'huître, la menace. Pourtant depuis le début de ce siècle, aux États-Unis, l'huître est reconnue pour sa place dans l'environnement et les services qu'elle prodigue pour la restauration de la biodiversité. Par sa capacité biologique à refléter les qualités de l'environnement où elle vit, l'huître est dite sentinelle* de l'environnement littoral.

Biologiste et ethnologue allons relater les voyages de l'huître dans les eaux françaises. Ses aventures sont d'autant plus intéressantes qu'elles dessinent une part des relations qui s'établissent entre l'humain et ce « mollusque marin pourvu d'une coquille*», mais aussi avec le littoral et le monde maritime.

\section{Descriptif biologique des huîtres plate et creuse}

Aborder les aventures des huîtres dans les eaux françaises, c'est d'abord découvrir qui est ce coquillage, connaître ses comportements biologiques et savoir si celle que l'on désigne toujours au singulier, l'huître, n'a réellement qu'une seule identité.

En réalité, depuis 150 ans en France, l'huître est plurielle. Ses identités, méconnues le plus souvent du consommateur, sont multiples: la plate* (Ostrea edulis); les creuses*: la portugaise* (Crassostrea angulata), la japonaise* (Crassostrea gigas); la triploïde... Ces coquillages ne sont pas vraiment maîtres de leur lieu de vie. Ils sont conditionnés par leur profil biologique; leur lieu de naissance puis de résidence; éventuellement leur état (sauvage/domestiqué/marchand...). La fabrication de leur destin par les univers professionnels ou le monde scientifique participe et peut aussi conditionner leurs identités.

\section{Balade avant sédentarité: l'huître en période de reproduction}

\section{Reproduction des creuses}

La reproduction des huîtres a lieu tous les ans, pendant l'été. Les huîtres creuses qu'elles soient portugaises ou japonaises, après la maturation sexuelle qui a lieu pendant le printemps et le début de l'été, expulsent dans l'eau les cellules reproductrices mâles 
(spermatozoïdes) et femelles (ovocytes). Après la fécondation, les oufs deviennent des larves qui sont soumises immédiatement aux mouvements des courants de marée. Quelques jours après, les larves munies d'une petite coquille naissante sont dites véligères*. Elles sont en effet munies d'un velum* ou voile qui leur permet à la fois de nager et de capter leur nourriture. Cette phase que l'on dit larvaire* dure environ trois semaines. À la fin de la phase larvaire, l'animal mesure un tiers de millimètre et est prêt à s'accrocher sur un support favorable. En plus du velum, un pied va permettre à la larve dite alors pédivéligère* de chercher son habitat définitif et de s'y fixer en secrétant un ciment. Ce nouveau mode de vie s'accompagne d'une métamorphose* le pied et le velum régressent puis disparaissent, des branchies et des palpes labiaux* se forment pour assurer la captation de la nourriture et son acheminement vers la bouche. La vie vagile* de l'huître n'aura donc duré que trois semaines et voici l'animal sessile*, accrochée à son support. Cet animal nouvellement fixé se nomme naissain*.

L'huître creuse ne vit pas n'importe où. Ne pas pouvoir se déplacer pour se nourrir nécessite de vivre dans des environnements où la nourriture est abondante. Les rochers, les estrans sableux ou vaseux des baies ou embouchures de fleuves et rivières répondent à cette contrainte. Ce sont des environnements variables, et pour compenser les fortes mortalités des trois premières semaines, les larves doivent être très nombreuses à la fécondation pour assurer le succès de la reproduction et la survie de l'espèce. Pendant trois semaines, les larves, malgré leur velum, sont dépendantes des courants littoraux et peuvent être portées plusieurs dizaines de kilomètres. Ceci confère à l'huître creuse une grande capacité à coloniser de nouveaux espaces bien que ce soit dans les sites les plus protégés que la reproduction est souvent la meilleure.

\section{Reproduction de la plate}

La reproduction de l'huître plate (Ostrea edulis) est sensiblement différente car la fécondation des ovocytes s'effectue dans l'huître, entre les valves et non pas à l'extérieur, dans l'environnement. Ainsi, l'huître plate garde-t-elle ses larves pendant une dizaine de jours avant de les expulser. Cette différence dans la stratégie de reproduction des deux espèces d'huîtres reflète aussi des habitats légèrement différents. L'huître plate vit dans un environnement légèrement plus profond et plus éloigné de la côte que l'huître creuse. Les variations de salinité y sont plus faibles et les fonds plus sableux, plus graveleux, ce qui donnera son nom à l'huître d'Arcachon, la gravette*.

Mais malgré ces différences, qu'il s'agisse de l'huître plate ou de l'huître creuse, les sites favorables sont souvent similaires et l'ostréiculture, dans son développement ne s'y est pas trompée, s'installant sur les sites connus du Bassin d'Arcachon, de 


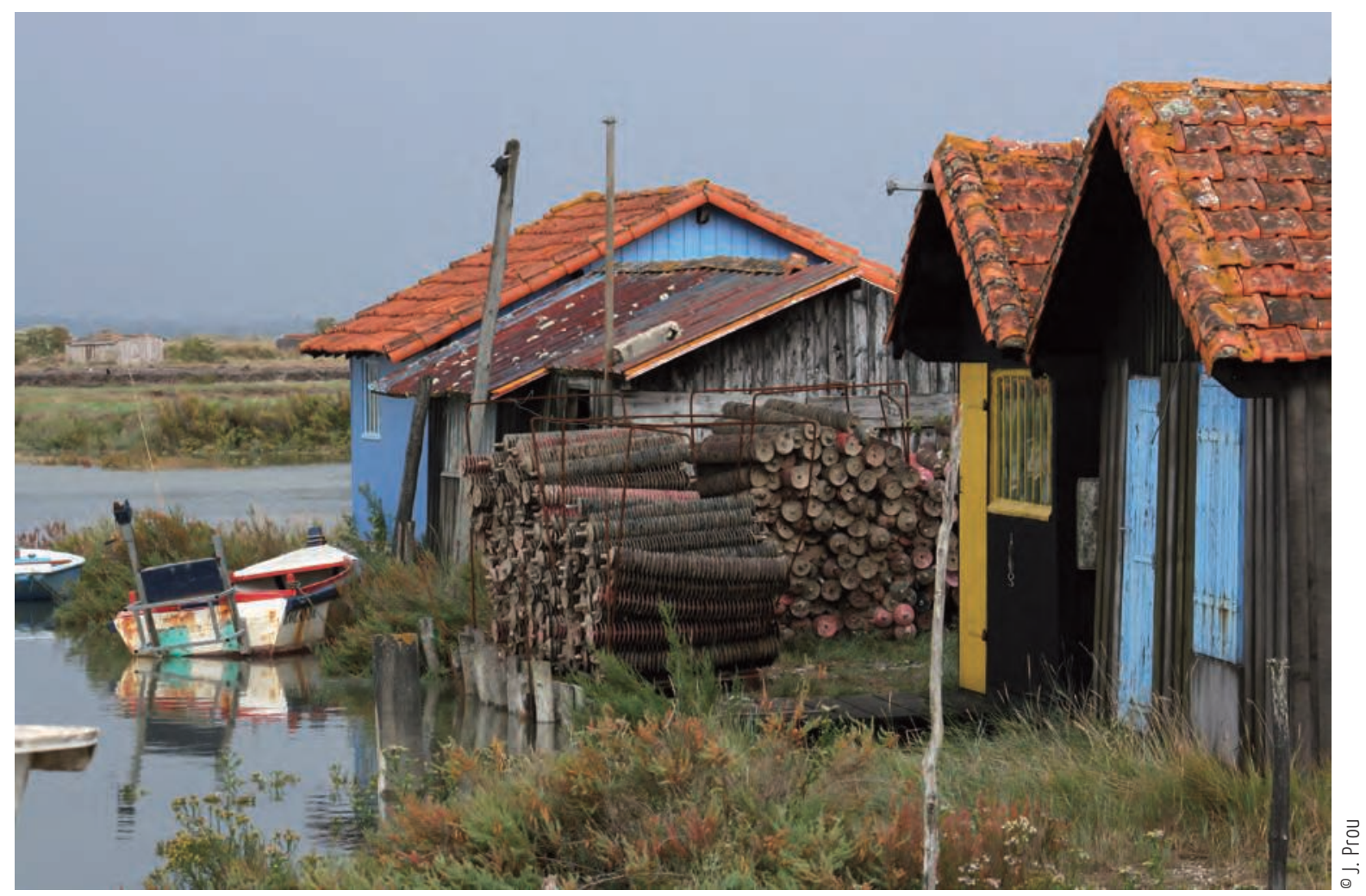

Cabanes ostréicoles à Chaillevette (17)

Les traditionnelles « cabanes » sont les lieux de travail à terre des ostréiculteurs et ostréicultrices. Proches du ponton pour l'amarrage du bateau, et entourées de matériels (ici des collecteurs de type « coupelle »), elles participent au paysage de l'ostréiculture. Le développement de l'élevage a encouragé progressivement la construction de bâtiments modernes et adaptés, laissant les cabanes traditionnelles pour des activités touristiques de loisirs. l'estuaire de la Gironde, de la Charente et des pertuis Charentais, des rias et abers bretons et des havres normands.

\section{Sessile ou vagile: avantages et inconvénients}

Pour se fixer, les huîtres ont besoin d'un substrat dur. Sur la côte, les rochers sont des supports favorables si leur exposition aux vagues et à la houle n'empêche pas la constitution de véritables gisements ou bancs naturels. Tout autre matériau est un support favorable à la fixation d'une nouvelle génération. Des collecteurs* ont ainsi été confectionnés pendant longtemps de bois, pierre, ardoise, fer, tuile... et aujourd'hui principalement de plastique.

Une autre coquille est aussi un support très favorable. C'est ainsi que se forment les bancs* d'huîtres, où les générations s'accrochent les unes aux autres. Ces bancs peuvent s'étendre sur plusieurs kilomètres et représentent de grandes quantités de géniteurs potentiels. Ces constructions, souvent assimilées à des récifs, participent à une bonne reproduction de l'espèce dans la mesure où l'expulsion des cellules reproductrices est souvent synchrone et donc favorise, quelques instants après la ponte et sur un site restreint, le succès de la fécondation. Lorsque les bancs d'huîtres sont exposés aux vagues et à la houle, il peut arriver que des coquillages s'en détachent et soient amenés jusqu'au rivage. Ces roulantes* ne peuvent néanmoins survivre très longtemps étant sans cesse soumises aux courants et aux vagues. 
Dans des environnements où la quantité de nourriture n'est pas limitée, le caractère sessile présente des avantages certains. Chez l'huître, les organes de locomotion sont quasiment absents (sauf le pied pendant la vie pélagique) au profit du développement, pendant la métamorphose, des branchies qui permettent outre la respiration, la prise de nourriture transportée par les courants. La motilité chez certains animaux pour la quête de nourriture est une source importante de dépense d'énergie. La sessilité de l'huître, au contraire, est synonyme de faibles dépenses énergétiques. Un des inconvénients de la sessilité est l'exposition de l'animal aux prédateurs et aux conditions environnementales défavorables. Comme beaucoup de mollusques à capacité de déplacement faible, la défense contre les prédateurs est matérialisée par la formation d'une coquille. Chez les mollusques bivalves, et chez l'huître en particulier, deux valves sont articulées et peuvent être refermées par un muscle adducteur* dès que les conditions extérieures deviennent défavorables. C'est le cas de l'attaque par des prédateurs ou de l'émersion qu'elle soit naturelle comme le mouvement des marées ou provoquée comme la pêche. Contrairement à la coquille Saint-Jacques dont la coquille n'est pas parfaitement étanche, mais qui peut se déplacer par fermeture rapide des valves, l'huître n'a aucun moyen de locomotion propre, mais elle peut s'isoler complètement en refermant ses valves. Ainsi, l'eau emprisonnée lui permet de vivre plusieurs jours sans s'ouvrir. L'absence de motilité devient alors paradoxalement, par le processus de protection, un élément qui favorise le transport.

\section{Voyages dans le temps d'un coquillage}

\section{L’huître fossilisée}

Un peu partout en Europe, les roches calcaires offrent des fossiles d'huîtres. Contrairement à de nombreux animaux qui ne résistent pas à la fossilisation, la coquille de l'huître ne se dégrade pas facilement et supporte sans grande transformation les enfouissements dans les sédiments marins et leur diagénèse*. Ainsi, les plus anciens fossiles d'huîtres remontent à plus de 200 millions d'années et on les retrouve dans le monde entier. À la mort de l'animal, les coquilles ont été enfouies dans des sédiments qui eux-mêmes ont été, avec le temps, transformés en roches, puis par les mouvements de l'écorce terrestre, remontés au-dessus du niveau de la mer. La présence d'huîtres fossilisées dans les roches

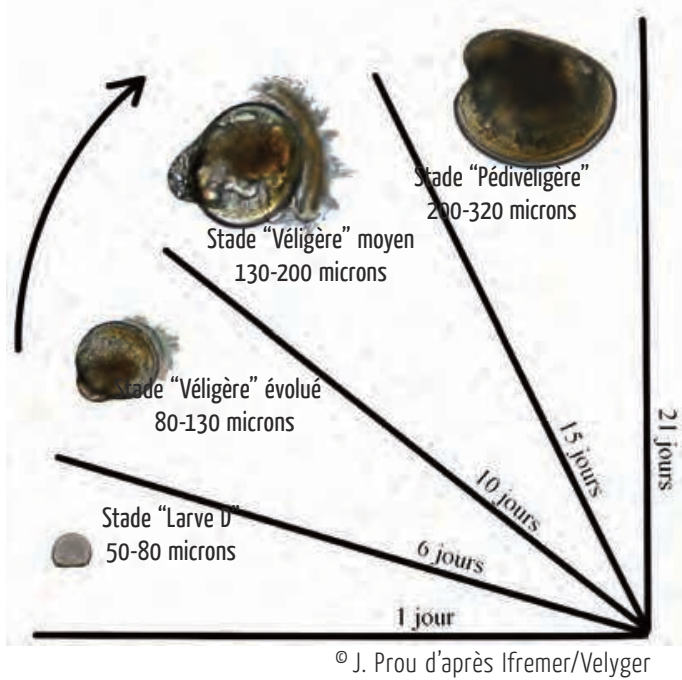

Évolution des larves de l'huître creuse,
Crassostrea gigas 


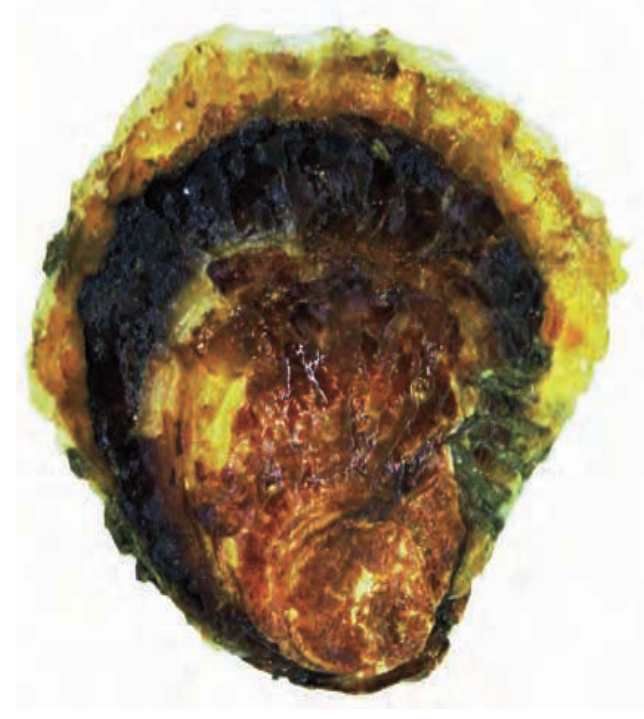

l'huître plate, Ostrea edulis est donc la marque d'un ancien rivage marin, ou de petits fonds, où les huîtres pouvaient se développer. Le genre Gryphaea, par exemple, est bien connu des paléontologues et c'est d'ailleurs en référence à ces fossiles que l'huître portugaise a été dénommée Gryphaea angulata avant d'être rebaptisée Crassostrea angulata. Ainsi, les huîtres voyagent aussi dans le temps, puisqu'elles offrent à l'homme, grâce à sa coquille, des marques temporelles de leurs passages.

\section{L'huître à la conquête du monde}

\section{Ostrea edulis vers les rivages de l'Europe}

En Europe, pendant la dernière glaciation, il y a 10000 ans, le niveau de la mer était inférieur de plus de 100 mètres à ce qu'il est actuellement. Au réchauffement qui l'a suivie, la fonte des glaces a provoqué une forte montée du niveau marin et, par suite du ralentissement du débit des rivières, une forte sédimentation a façonné le relief des côtes actuelles, en particulier les larges estrans sablo-vaseux des estuaires comme la Gironde, la Loire, la Seine, les rivières et rias bretonnes, les pertuis Charentais, etc. Lors de l'élévation du niveau de la mer, de larges espaces littoraux ont été recouverts par la mer. Transformées en estran car se découvrant à chaque marée, ces zones maritimes sont riches en particules organiques et en plancton. L'huître plate Ostrea edulis va suivre cette évolution du rivage et s'installer préférentiellement au niveau de la limite de la basse mer. L'huître plate, espèce endémique des côtes européennes, va devenir ressource alimentaire dès la période préhistorique (Gutiérrez-Zugasti et al. 2011).

\section{La plate (Ostrea edulis) : l'autochtone des eaux européennes}

La plate est la seule huître autochtone du sud au nord de l'Europe depuis plus de 10000 ans. Ses premiers voyages hors du littoral comme aliment et produit marchand dateraient, dit-on, de l'époque romaine. Cette denrée, prisée par la haute société romaine, aurait même fait l'objet de publicité autour du Lac Lucrin, près de Naples. Les vases retrouvés dans cette région font en effet cas de décorations mettant en scène des éléments montrant que les huîtres n'étaient pas simplement pêchées, mais sans doute aussi affinées avant d'être consommées.

Les Romains étaient friands d'huîtres. Les textes littéraires (Pline l'Ancien, Sénèque, Ausone, etc.) relatent que l'ensemble de l'empire s'échangeait des connaissances et des informations sur la qualité des huîtres de L'Hellespont, d'Angleterre, de Galice, etc. La notion de terroir était déjà en place, prête à qualifier le lien étroit entre l'huître et son environnement. En effet, l'huître est un filtreur. Sa chair est constituée à $80 \%$ d'eau. Une huître adulte peut filtrer jusqu'à 100 litres d'eau de mer par jour. Plancton en suspension dans l'eau ou vivant sur les sédiments, particules organiques associées aux sables et vases et remises en suspension par les courants: chaque écosystème 
va offrir aux huîtres un cocktail d'éléments nutritionnels qui dépendra de la forme des côtes, de la profondeur, des sédiments, des rivières et fleuves ou du climat. Le lien entre le milieu où elle se nourrit et cette chair est présenté comme majeur. À l'époque romaine, les huîtres vont voyager accompagnées d'un discours gustatif sur leurs origines. Elles sont en quelque sorte, les témoins de la géographie de l'Europe à l'époque romaine.

Contrairement aux poissons frais, la possibilité de transporter des huîtres vivantes sur de grandes distances a aussi nourri ces témoignages gastronomiques. Leurs déplacements pouvaient se faire par voies terrestres autant que dans des bateauxviviers permettant de véhiculer ces coquillages sur de grandes distances. Les huîtres étaient ainsi consommées du nord au sud de la France, en Germanie, en Autriche... Le transport de l'huître est facilité du fait que deux valves hermétiquement fermées conservent son eau de mer et protègent sa chair. Des fouilles archéologiques ont d'ailleurs montré que, avant de les consommer, les Romains savaient les conserver loin de leur lieu de pêche en les entreposant dans des bassins d'eau salée (Barbier, 1886). Les amas de coquilles témoignent de ce savoir-faire. En France, des écrits relatent que les voyages de ce coquillage ont perduré au Moyen Âge et au cours des siècles suivants. Certains rois de France en mangeaient de grosses quantités.

Les Romains avaient peut-être observé que naturellement l'huître se maintient fermée durant l'exondation des basses mers et ont-ils tenté de reproduire ce phénomène pour faciliter son transport sur de longues distances. Dans tous les cas, depuis fort longtemps, dans les eaux françaises, la pratique est d'éduquer* l'huître pour qu'elle demeure coite* pendant le voyage (Legué 2004). Cette opération appelée aussi trompage* facilite son transport. Les huîtres peuvent ainsi rester vivantes près de deux semaines à condition qu'elles soient empaquetées bien serrées les unes contre les autres et dans un endroit frais.

\section{Nomadisme d'un coquillage sédentaire: Les aventures des huitres dans les eaux françaises depuis 150 ans}

Même au temps des Romains, les populations côtières n'avaient pas besoin d'être aisées pour pêcher et consommer des coquillages et en particulier des huîtres. Mais c'est avec la demande croissante venant des villes que le transport des huîtres vers l'intérieur des terres s'est intensifié. Le coquillage, mangé vivant, saumuré ou cuisiné en ragoût a permis de développer ce marché. De la fin de l'Empire romain jusqu'à la naissance de l'ostréiculture aux alentours des années 1860, la pression sur les bancs naturels d'huîtres fut très forte. Deux lois, l'édit de Moulins (1566) puis l'ordonnance de Colbert de 1681, en posant le principe d'inaliénabilité du Domaine Public Maritime, tentèrent de juguler le pillage des ressources qu'offraient les huîtres des bancs naturels. Mais les contrôles demeurèrent impuissants.

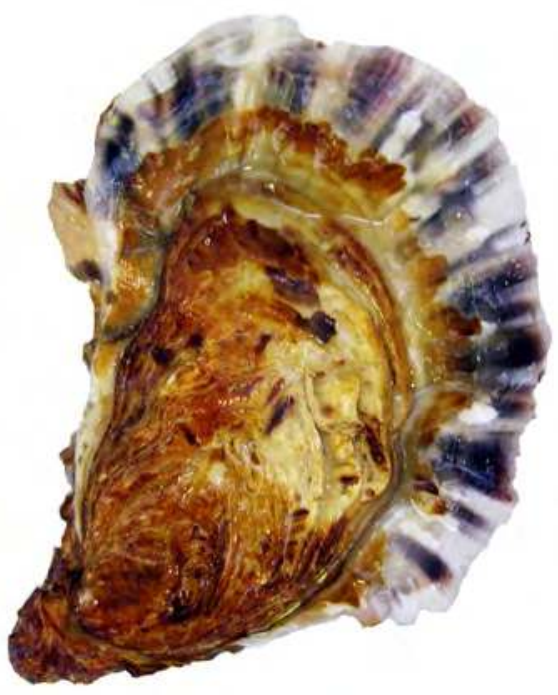

L’huître creuse, Crassostrea gigas ou angulata 


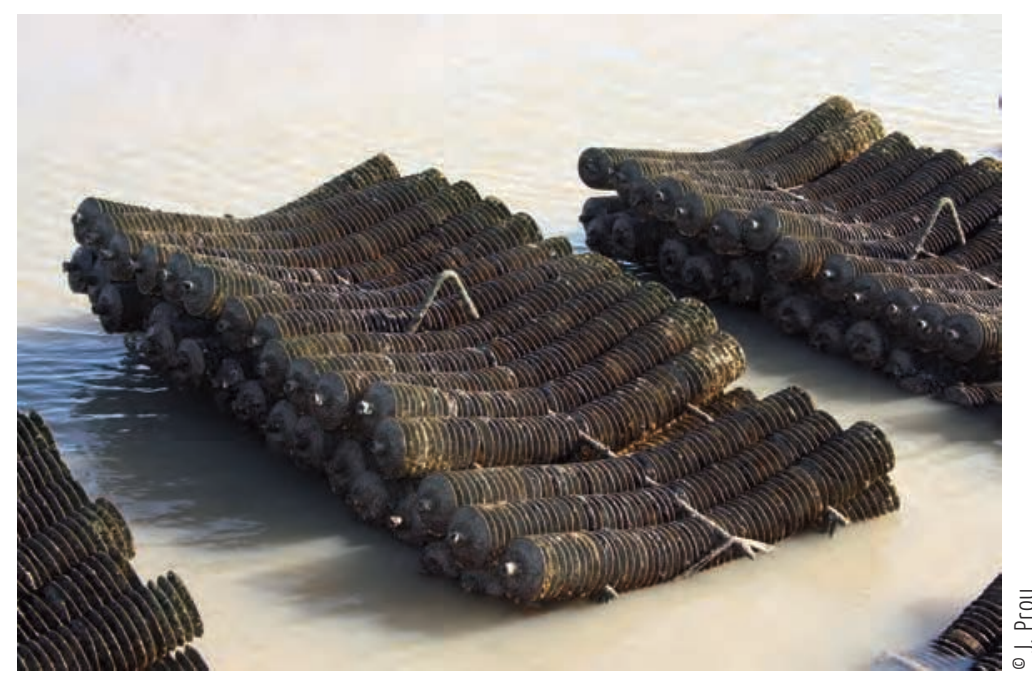

Collecteurs (type « coupelle ») utilisés pour le captage du naissain

Placés au début de l'été sur les estrans, les collecteurs sont des supports pour les larves d'huitres. « À la fin de la phase larvaire, l'animal mesure un tiers de millimètre; il est prêt à s'accrocher sur un support favorable ».

\section{Voyage et disparition de l'huitre indigène: la plate}

L'activité ostréicole ne fut jusqu'au milieu du XIx siècle qu'une activité de cueillette et de pêche. Les huîtres étaient draguées ou ramassées à pied sur les gisements naturels et « jetées pêle-mêle sans aucune précaution » (Diderot 1765) à l'engraissement dans des bassins aménagés sur la partie haute de l'estran. À cette moitié du XIX siècle, les bancs naturels d'huîtres plates en France étaient dans l'incapacité de satisfaire la demande de consommation.

Néanmoins, pour répondre à cette consommation, mais aussi parer la fragilisation des bancs d'huîtres plates, à Arcachon et en Bretagne, une autre activité va se développer: l'ostréiculture*. Ce passage de la cueillette à la culture fut marqué par une transformation technique majeure: le captage* ou collectage* des larves d'huîtres sur collecteurs artificiels.

Deux hommes en furent les principaux initiateurs ${ }^{1}$. Victor Coste (1861), savant naturaliste et M. De Bon, commissaire de la marine à Saint-Servan. Le premier, à la demande de l'empereur Napoléon III, rendit public en 1855 le récit de son « voyage d'exploration » en Italie et ses recommandations concernant la reproduction des huîtres. Son enthousiasme et l'appui de l'empereur permirent de conforter les résultats obtenus par De Bon dans le repeuplement des huîtrières* de la Rance, près de Saint-Malo.

Dans le domaine de la reproduction des huîtres, Coste prodigua ses conseils à Saint Brieuc, à Arcachon, dans les pertuis Charentais, en Méditerranée. Il y proposa de réserver des zones où l'administration maritime organisera la collecte des larves afin de reconstituer les bancs naturels dévastés. Le captage de l'huître plate s'effectuait par la mise en place de divers planchers en bois susceptibles de recueillir le naissain. Ces essais eurent des fortunes diverses.

À Saint-Brieuc, après de premiers essais concluants, les bancs naturels se développèrent mais sans doute sous la double pression de la demande en huîtres non satisfaite et des dragages répétés des pêcheurs pour la rogue*, ces bancs furent à nouveau décimés. À Arcachon, des parcs impériaux* furent créés et gérés par l'administration. Là aussi, le succès fut au rendez-vous dès 1862 et des associations entre marins et investisseurs permirent la concession de parcs à huîtres où le captage s'effectuait, alimenté par les pontes des géniteurs des parcs impériaux. L'ostréiculture était née, le captage artificiel ayant été progressivement adopté par les huîtriers* des côtes de France.

Pour pallier la mortalité croissante de la plate charentaise, la Marennes*, la destruction de ses bancs naturels et les nouvelles méthodes ne suffisant ni à satisfaire la consommation ni à repeupler les huîtrières, une marchandisation du coquillage entre professionnels va s'instaurer.

Les huitriers arcachonnais vont livrer par bateau des tonnages d'huîtres aux Charentais qui, à partir du milieu du xix siècle, les affineront dans leurs claires*. Les réseaux d'échange avec la Bretagne s'établirent plus difficilement, d'une part en raison d'une plus grande distance, 
en second lieu parce que la production artificielle se développa sur les côtes bretonnes plus tardivement qu'à Arcachon. Ce furent d'abord les huîtres pêchées sur gisements qui furent livrées aux Marennais. Cette pêche à la drague était réglementée et n'était autorisée par l'Administration que quelques jours par an.

La dépendance du monde ostréicole charentais à l'égard des autres bassins était atténuée par la concurrence à laquelle Arcachonnais et Bretons se livraient dans la domination de ce marché. Une certaine cohésion sociale apparut à ce moment-là au sein des huîtriers charentais, suscitée par une difficulté commune à s'approvisionner en huîtres, et par une opposition farouche à

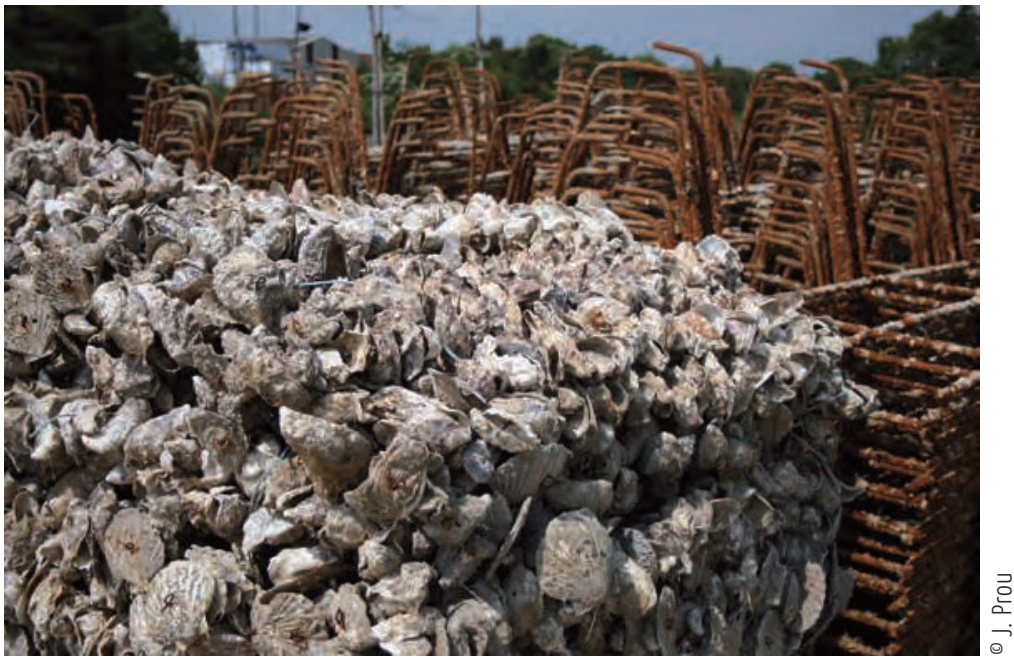
toute ingérence étrangère au sein de leur territoire. Cependant, l'obligation de se fournir en huîtres contraint ces professionnels à migrer temporairement vers les bassins producteurs de Bretagne et d'Arcachon. Ils élargirent alors leurs réseaux de relations sociales auprès de communautés ostréicoles étrangères (Bretagne et Arcachon) tout en s'opposant à une intrusion de ces derniers dans leur espace professionnel. Ils ne purent toutefois résister à une pénétration culturelle résultant de l'intercommunication entre ces groupes. Ainsi, même s'ils ont fort peu pratiqué l'ostréiculture avec la plate, les Charentais ont adopté le captage des larves d'huîtres sur collecteurs artificiels avec la portugaise.

Avec le développement du captage à partir des années 1860, la production d'huîtres s'intensifiant, il fut indispensable de faire parvenir le produit au lieu de consommation dans les plus brefs délais. Si le développement du transport ferroviaire attira les populations citadines vers les littoraux, il eut pour autre fonction de faciliter le transport des huîtres vers les lieux de consommation. En Charente-Maritime, jusqu'en 1945, 90 \% des huîtres marchandes étaient livrées par voies ferrées.

En France, les bancs naturels d'huîtres plates furent régulièrement décimés sur les côtes méditerranéennes, normandes et du Nord bien avant le xIx ${ }^{e}$ siècle. Elles disparurent progressivement des bassins d'Arcachon et de Charente-Inférieure (Charente-Maritime), principalement vers la fin du xix /début du xxe siècle. Les eaux bretonnes la « retiendront » jusque dans les années 1970, période à laquelle elle fut fragilisée par des épizooties. Depuis, elle survit sur les côtes bretonnes, et selon le récit que quelques ostréiculteurs français relatent, la plate originaire des eaux du nord de l'Europe (anglaises, irlandaises...) « vole ou navigue » aujourd'hui au secours de celle couramment dénommée la Belon*.

\section{La portugaise: une migrante sédentarisée}

Si le bassin d'Arcachon invente l'ostréiculture avec la plate, cette profession prend réellement forme au xxe siècle dans ce bassin et en Charente-Maritime avec une autre huître: la portugaise.

L’aventure européenne de cette huître débuta, a-t-on supposé, au Xvi siècle, à l'époque des grandes découvertes, dans les eaux des mers orientales de Taïwan (Lapègue et al. 2004). 


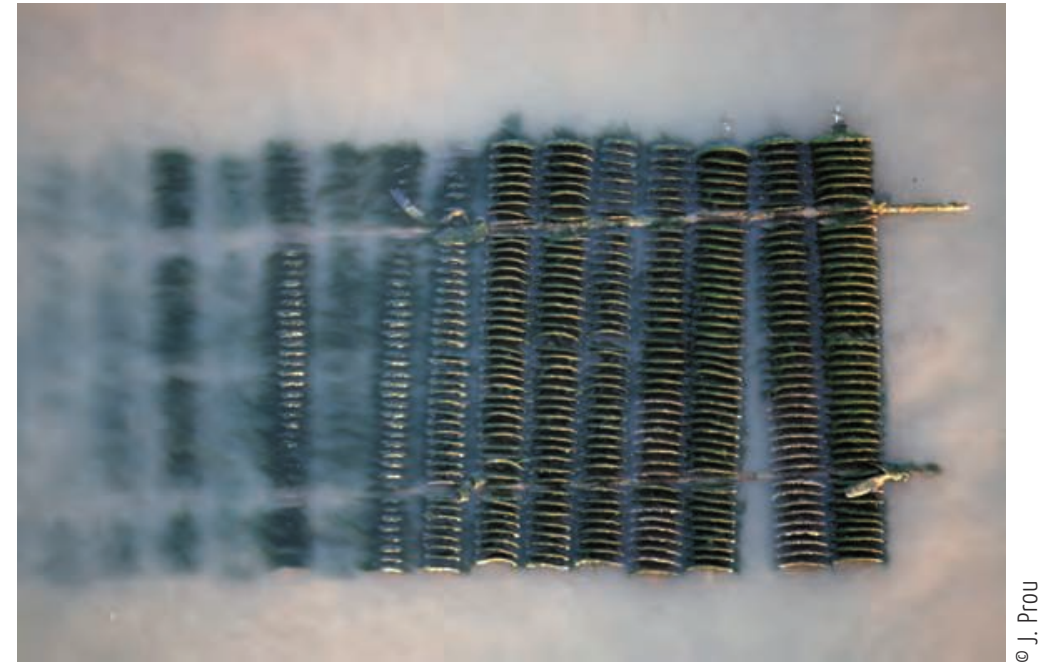

Après la fécondation et la phase larvaire, les larves d'huitres s'accrochent aux collecteurs posés par les ostréiculteurs. Quelques mois plus tard, les naissains sont détachés par vibration du collecteur de type « coupelle ». Cette opération dite de détroquage permet la suite de l'élevage en poches, début des voyages que l'ostréiculteur impose à l'huître.
Fixée aux coques des bateaux, notre huître voyagea vers le Portugal où elle s'implanta naturellement, ayant trouvé des conditions environnementales favorables (température de l'eau, estran sablo-vaseux, apports d'eau douce, baie semi-fermée). L'homme, à ce que le mythe d'origine véhicule, lui fit parcourir la seconde partie de son trajet vers la France. En effet, l'augmentation de la consommation des huîtres en France, les aléas climatiques de 1867 et 1868 conjugués à l'action du bigorneau perceur, prédateur de l'huître, ont diminué l'offre.

Dès lors, de la fin 1866 à mai 1868 (Barboza du Bocage 1868), treize rotations de navires français rapatrièrent vers Arcachon plus de 12 millions de portugaises depuis l'estuaire du Tage, près de Lisbonne. Lors de la dernière rotation, en mai 1868, un bateau à vapeur, le Morlaisien, participait au transport des huîtres du Portugal vers Arcachon. Peu avant d'entrer dans le port français, une tempête se leva qui contraint le capitaine, Hector Patoiseau, originaire du Château d'Oléron (Charente-Maritime), à se réfugier dans l'embouchure de la Gironde où il demeura ancré plusieurs jours. Du fait de cette attente, la cargaison s'abîma et le capitaine reçut l'ordre de gagner le large pour rejeter en mer son chargement avarié. Incompréhension ou faute, il procéda en fait à l'opération près de la côte. Quelques coquillages encore vivants trouvèrent là un milieu favorable à leur développement et furent si prolifiques qu'ils formèrent bientôt de véritables gisements. La portugaise entama ensuite naturellement, au fil des courants, la troisième étape de son périple et progressa jusqu'au Havre du Payré (Vendée) qu'elle atteint en 1879, non sans avoir pénétré le bassin charentais de l'estuaire de la Seudre et celui de la Charente (Papy 1941). En huit générations de portugaise (1867-1874), les bancs naturels de cette nouvelle ressource se développèrent au point d'assurer une exploitation commerciale. Dès 1874, ce coquillage aurait été livré à la consommation (Champouillon 1876).

Tout d'abord rejetée par les huîtriers de la rive gauche de la Seudre, affineurs d'huîtres plates, l'huître portugaise fut accueillie comme une manne par une multitude d'individus de pauvre condition: marins, journaliers, paysans. Ceux-ci furent à l'origine d'un nouveau groupe professionnel ostréicole en adoptant les techniques de captage: les ostréiculteurs. Ils s'établirent sur la rive droite de la Seudre, rive opposée à celle des affineurs de plates. «C'était l'eldorado » disaient les ostréiculteurs qui ont vécu cette période.

Une résistance opiniâtre à l'huître allogène, « les encombrantes portugaises », fut alors engagée par les affineurs, exploitants d'huîtres « indigènes » (Hervé 1935), les Marennes,

« Opposons une barrière énergique à ce flot immonde, déclarons une guerre d'extermination à l'huître du Portugal, laissons piller les bancs qu'elle a envahis, que partout elle soit traquée sans relâche » (Dr Leroux in Grelon 1978).

Ce conflit cultural fut suscité par la crainte des huîtriers de voir l'espace de reproduction de la plate envahi par la portugaise. Les chercheurs de l'époque notent que les deux 
espèces Ostrea edulis et Gryphaea angulata ne peuvent pas s'hybrider (Bouchon-Brandely, 1882), qu'elles n'ont pas les mêmes modes de reproduction et qu'elles n'occupent pas les mêmes sites sur le littoral. La forme arrondie de la valve inférieure de l'huître portugaise est plus adaptée aux sédiments meubles de l'estran découvrant. L'huître plate y meurt car s'enfouissant dans les vases (Fischer 1880, Leroux 1882).

Si l'huître plate peut se reproduire dans les eaux plus froides du nord de l'Europe, l'huître portugaise n'a pu se reproduire qu'exceptionnellement au nord de la Loire car les températures y sont trop basses pour assurer la maturation des géniteurs. Grâce à la portugaise, le bassin d'Arcachon, l'estuaire de la Gironde et les pertuis Charentais devinrent des bassins naisseurs* de portugaises laissant les Bretons s'approprier progressivement l'huître plate ${ }^{2}$.

Cette distinction biologique entre les deux espèces s'imposa aussi dans les champs économiques et culturels et prit la forme d'un double antagonisme:

- l'un socio-économique, se traduisit sur le plan local par une distinction entre "l'Huître du riche, la noble Marennes" (termes orthographiés avec une majuscule dans les ouvrages du xxe siècle) et l'huître du pauvre, la portugaise, produit de la fange, dont l'huîtrier disait qu'elle conservait « le goût de la vase sur laquelle elle vit ». Chacune de ces espèces avait son territoire, le marais pour la plate, l'estran pour la portugaise. À chacune était attaché un univers social différent, rival même, qui faisait se confronter les groupes des deux rives de la Seudre;

— le second culturel. En effet, en Charente-Inférieure, mais aussi à Arcachon, l'ostréiculture prend réellement place avec la portugaise.

En France, l'identité de la portugaise est strictement régionale, arcachonnaise ou charentaise car la fraîcheur des eaux au-delà de la Vilaine (entre Loire Atlantique et Morbihan) n'incite pas ses larves à naviguer vers la Bretagne. Ses trois années de vie avant commercialisation se feront exclusivement dans le bassin où elle est née. L'ostréiculteur la faisant caboter régulièrement entre l'estran, l'établissement, éventuellement le marais à Marennes-Oléron*.

\section{La japonaise: la bourlingueuse, objet de transhumance}

Si l'introduction de Crassostrea angulata, l'huître portugaise, ne fit pas l'objet, dès le départ, d'une volonté de transplantation géographique d'une espèce, les introductions de Crassostrea gigas au début du siècle sur la côte ouest des États-Unis (Steele, 1964) et dans les années 1960-1970 en France furent des implantations volontaires et organisées.

Peu avant la disparition de la portugaise en 1971, une nouvelle huître va connaître les eaux françaises: la japonaise. Dans les années 1960, des mortalités de
Sur l'estran, poches d'huîtres alignées sur des tables recouvertes à marée haute. Une huître adulte peut filtrer jusqu'à 100 litres d'eau de mer par jour.

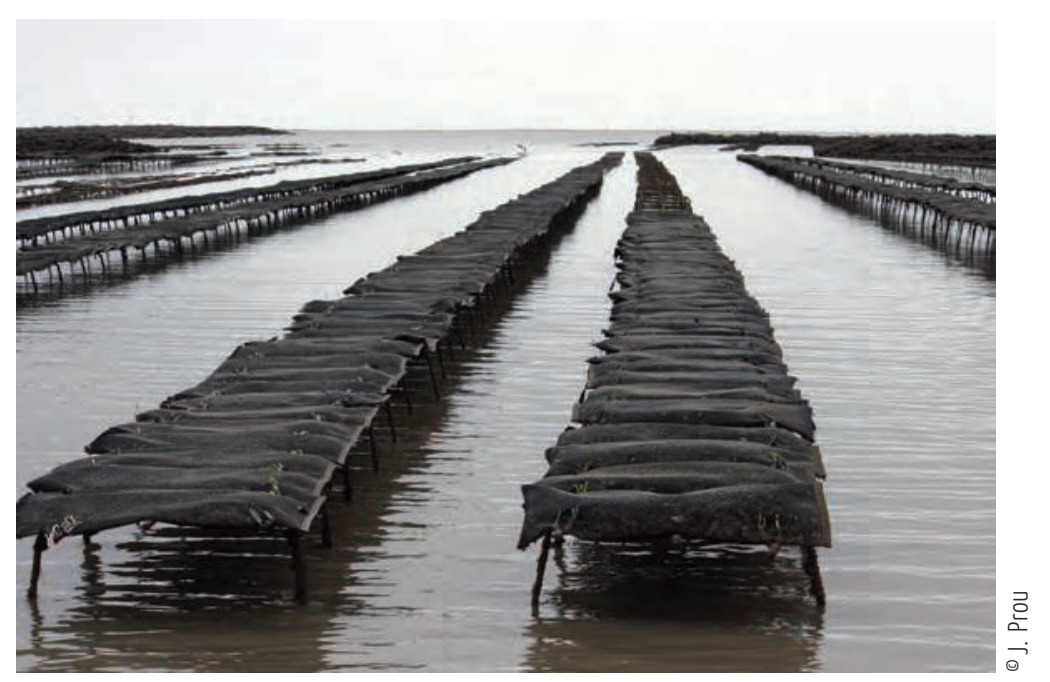




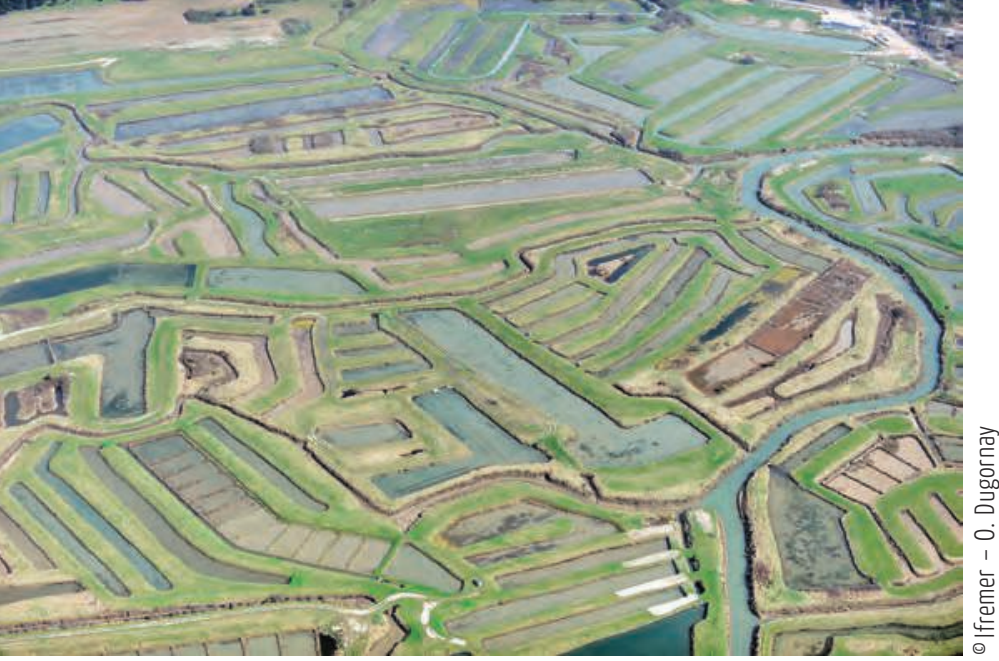

Anciens marais salants transformés au milieu du xixe siècle en marais de claires ayant pour fonction l'affinage des huîtres

Les claires sont un élément typique des paysages de l'ostréiculture charentaise.

Carte des mouvements par la route des naissains d'huîtres japonaises (Crassostrea gigas) des sites de captage vers les sites d'élevage français

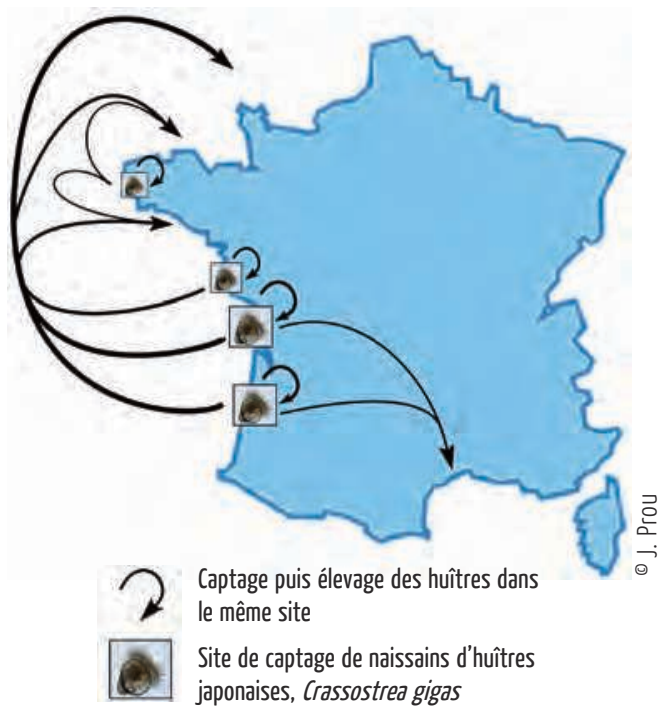
meilleure élève*. l'huître du Portugal et de mauvaises performances de croissance ont amené les producteurs français à rechercher une espèce de remplacement. Dès janvier 1966, un producteur des pertuis Charentais obtient une autorisation d'importer des huîtres Crassostrea gigas de moins de cinq centimètres venant de la région de Sendaï, dans la préfecture de Myagi, au Japon. Après s'être assurés que les huîtres importées ne présentaient pas les mêmes symptômes que les huîtres portugaises et qu'un ver parasite pouvait être éliminé par retrempage dans de l'eau douce, les pouvoirs publics autorisèrent les importations du Japon (sous la forme de naissains) et aussi de la Colombie Britannique (Canada) sous la forme d'huîtres adultes. Ainsi de 1971 à 1977, des importations privées furent complétées par une action publique de reconstitution des stocks de géniteurs: l'opération « resur». En 1975, alors que l'huître portugaise avait pratiquement disparu à cause des maladies, les premiers naissains de Crassostrea gigas furent collectés et montrèrent une croissance et une résistance aux maladies compatibles avec le renouveau de l'activité ostréicole. L'ostréiculture connut avec la japonaise des transformations sociales, techniques, mais aussi spatiales majeures. Au cours des trois années nécessaires à la production d'une huître marchande, les diverses opérations se maintiendront dans les bassins d'origine (élevage, affinage pour Marennes-Oléron, commercialisation). Toutefois, les parcs bretons étant libres d'occupation du fait de la mortalité de la plate, et l'estran normand étant vierge de toute culture ostréicole, quelques ostréiculteurs producteurs de naissain gagnent ces territoires plus riches en phytoplancton que les eaux de leurs bassins, et invitent la japonaise au voyage afin d'en faire une

Nombre d'ostréiculteurs des bassins d'Arcachon et de Marennes, jusqu'alors uniquement marins-paysans* et dont l'activité et l'identité étaient strictement régionales, deviennent aussi camionneurs. Ils pratiquent la transhumance vers les eaux bretonnes et normandes, calant le rythme de leurs déplacements sur le cycle lunaire des marées de vives-eaux (les malines*). Ainsi, toutes les deux semaines, des tonnes d'huîtres pratiquent des formes de transhumance parcourant en camion les routes des littoraux français. Puis après engraissement dans des eaux nourrissantes de Bretagne et de Normandie, à chacune de leurs phases de croissance, elles rentrent «à domicile » (Arcachon, MarennesOléron), ou pour être affinées (Marennes-Oléron), et surtout pour être vendues sous l'appellation locale. Au cours des trois années nécessaires pour produire une huître marchande, ce coquillage peut parcourir cinq fois les routes françaises entre son lieu de naissance (Arcachon, Marennes-Oléron), d'élevage (Bretagne Sud ou Nord, Normandie, étang de Thau), de commercialisation (marchés, commerces, grossistes, Rungis) et enfin de consommation (restaurants, domiciles). 
L'époque où les Charentais initiaient les techniques d'élevage de la japonaise aux Bretons et aux Normands est actuellement révolue. Ce rôle leur conférait un certain pouvoir auprès des populations indigènes. L'apprenti d'hier, Breton ou Normand, détient maintenant, au moins sur l'estran, l'art et la manière du maître. La domination du magister aurait pu être conservée avec la creuse, Arcachon et Marennes-Oléron étant les seuls lieux de production de naissain, ils n'ont su la préserver, n'ayant pas déposé une Appellation d'Origine Protégée (AOP)*. Hormis pour le naissain, les professionnels de Bretagne, Normandie ou même de l'étang de Thau (Languedoc-Roussillon) tendent à s'affranchir de toutes dépendances à l'égard des bassins du sud de l'Atlantique. Ils sédentarisent la japonaise dans leurs bassins pendant sa période d'élevage. Lors de sa commercialisation, aucune appellation n'évoquera ses éventuelles origines.

Deux autres raisons ont aussi favorisé l'affranchissement des Bretons et des Normands par rapport aux bassins producteurs de naissain:

- La colonisation par la japonaise d'espaces dont la température des eaux, les stocks de géniteurs et les conditions de courants sont favorables à sa reproduction. Ainsi, en France, la rade de Brest et d'autres secteurs de Bretagne Sud et Vendée, qui ne connaissaient qu'un captage limité les années favorables sont maintenant en état de soutenir l'ostréiculture bretonne par la fourniture de naissains. La Wadden See (néerlandaise et allemande) a été colonisée dès 1980 (Diederich et al. 2005, Schmidt et al. 2008). En 2010, le Danemark, la Suède et la Norvège (Wrange et al. 2010) voient des populations de Crassostrea gigas s'installer. Dans ces pays, cette espèce est considérée comme invasive.

— Une « révolution » avec l'émergence d'un nouveau métier : l'écloseur*. Cette profession maîtrise la reproduction de l'huître en dehors de son milieu naturel et l'élève dans des bassins jusqu'à la taille de $3 \mathrm{~mm}$. Au-delà, le nurseur prend le relais et l'amènera jusqu'à la taille demandée par l'ostréiculteur entre $3 \mathrm{~mm}$ et $3 \mathrm{~cm}$. Ainsi, la phase larvaire des huîtres nées en écloserie s'effectue à terre dans des bacs alimentés en eau de mer. Les larves ne connaissent pas, comme leurs homologues issus du captage en milieu naturel, les conditions du milieu extérieur. Elles ne connaissent pas non plus le collecteur puisque leur captage s'effectue sur des grains de sable afin de fournir à l'acheteur un produit « une à une », c'est-à-dire dans lequel les huîtres sont détachées les unes des autres. Ainsi, les sites bretons, normands ou méditerranéens ne dépendent plus exclusivement des sites arcachonnais et charentais. De plus, ces écloseries-nurseries ont bénéficié dès les années 2000 d'un résultat de la recherche scientifique: la polyploïdisation* des huîtres. Les mortalités estivales récurrentes depuis la fin des années 1980 expliqueraient l'essor de l'huître triploïde selon des professionnels interrogés. 


\section{L'abandon du sauvage et de la domestication au profit de l'artificiel et du « scientifique ». La triploïde ou la fin du voyage de l'ostréi-culture?}

Produit sauvage, naturel pendant quelques milliers d'années, l'huître est, depuis l'émergence du captage, coquillage culturel. En France, son histoire éthologique se confond avec celle de l'ostréiculture. La dynamique de cette communauté est ancrée dans l'aventure de ce coquillage. L'huître et l'ostréiculture sont assujetties l'une à l'autre, leurs évolutions sont simultanées. Ce coquillage est pour ceux qui en vivent une tradition alors même que ce passé est récent et manque de véritables fonds culturels (aucune fête, aucun saint, ni la moindre croyance ou légende spécifiques à cette communauté ostréicole). L'huître incarne à elle seule une culture dans laquelle les individus qui en vivent viennent puiser leur identité.

Reproduction et croissance demeuraient jusqu'à il y a peu, naturelles, presque indépendantes de toute intervention humaine, de tout apport de substances artificielles.

Avec une offre de naissains sélectionnés ${ }^{3}$, les écloseries ont fondamentalement changé le paysage de l'ostréiculture. Qu'ils soient triploïdes ou diploïdes, une sélection est opérée et le mode de reproduction et d'élevage des premières semaines (nursage) n'a plus grand-chose à voir avec la reproduction en milieu naturel où les pontes de cellules mâles et femelles se rencontrent au gré de la position des stocks de géniteurs et des courants, et où la larve « prête à se fixer » va choisir son collecteur.

L'huître triploïde du fait de son matériel génétique et ses modes de reproductions n'est plus sauvage, ni même produit de culture. Depuis sa transformation, «l'usage du monde ${ }^{4} \gg$ de ce coquillage tend à se restreindre. En écloserie, la larve d'huître abandonne son voyage initiatique avant de devenir coquillage. Le captage de l'huître n'a plus raison d'être. Dans certains secteurs ostréicoles, la triploïde semble ne plus migrer ni même transhumer, sa seule excursion se limitant au « voyage » qu'elle fait en camion entre l'écloserie et son unique lieu de résidence: le bassin dont elle porte le nom. L'exploitant de ce coquillage n'est plus qu'éleveur. Il abandonne dès lors son statut d'ostréiculteur.

\section{Une dernière fragilité ou un rebond}

Depuis 2008, de fortes mortalités touchent les naissains d'un an. Pour pallier le manque d'huîtres résultant des taux de mortalité de 80 \%, les professionnels ont d'une part, augmenté le nombre de collecteurs et d'autre part eu recours au naissain vendu par les écloseries 5 .

Mais l'adhésion individuelle des professionnels à la triploïde ne doit pas faire oublier l'attachement collectif de la profession à un recours au naissain issu du captage dans le milieu naturel. Les mortalités ont ainsi incité les pouvoirs publics et la profession à mettre en place un plan d'ensemencement des bancs naturels avec des géniteurs sélectionnés. Il s'agit, à l'aide d'une démarche de sélection génétique, de reconstituer des bancs naturels susceptibles de fournir du naissain libre de droit et sans coût autre que celui du collecteur et de sa pose.

D’autre part, le caractère contagieux des mortalités a été observé par les professionnels et prouvé par les scientifiques. La Commission européenne ${ }^{6}$ et l'État français ${ }^{7}$ ont pris en compte cette caractéristique en légiférant sur la gestion du risque de propagation des maladies et donc sur les voyages de l'huître entre sites. 


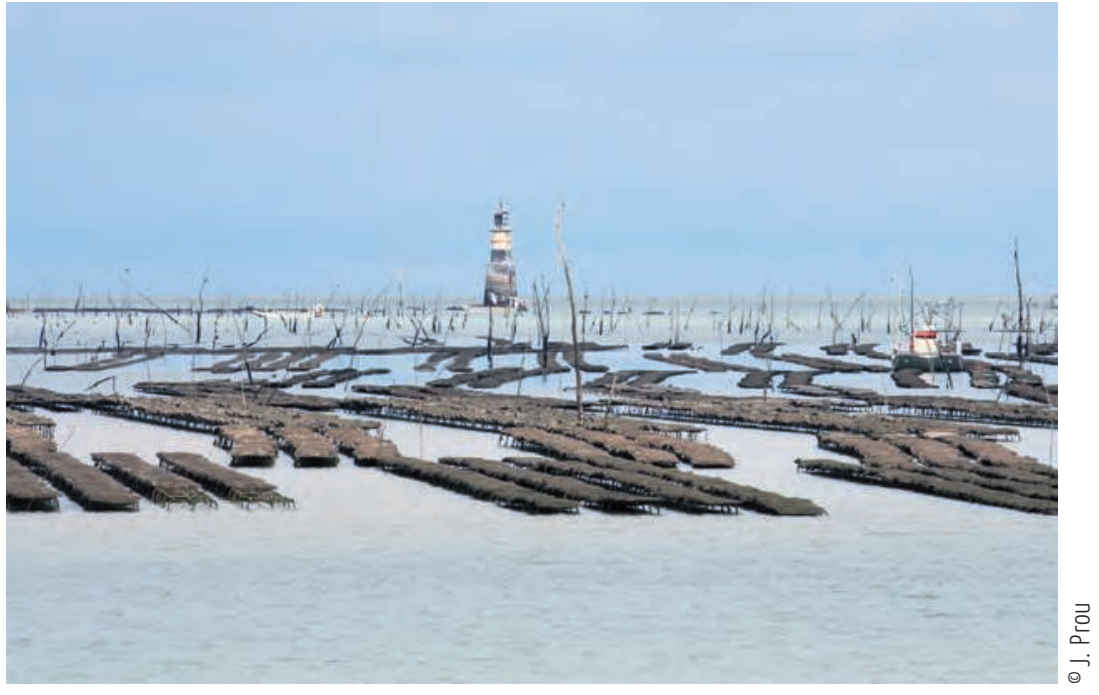

Les parcs à huîtres et leurs balisage offrent des éléments de paysage très particuliers. Réglementée dans chaque département par le Schéma des Structures des Exploitations de Cultures Marines, l'ostréiculture est un des acteurs principaux du domaine littoral par son implantation ancienne sur le Domaine Public Maritime.

La mise en place de parcs naturels marins, les évaluations environnementales liées aux plans d'aménagement du Domaine Public Maritime ${ }^{8}$, forcent à la définition d'une ostréiculture durable. Dans le même temps, le monde ostréicole, mortalité oblige, redécouvre les ressources de la pêche des bancs naturels ${ }^{9}$ et leurs influences dans les écosystèmes littoraux.

En Charente-Maritime, depuis les années 90, quelques bancs de plates sont réapparus de façon naturelle. Draguées par bateaux, elles sont commercialisées directement. La recomposition naturelle de ces gisements a une valeur environnementale manifeste; on peut néanmoins se demander pourquoi jusqu'alors, pour les préserver, ils n’ont jamais fait l'objet de mesures de gestion par les instances officielles (Affaires Maritimes, Ifremer...).

En outre, des mouvements de consommateurs, de défense et de protection de l'environnement, et aussi d'associations d'ostréiculteurs, remettent en cause les évolutions récentes d'une forme d'ostréiculture artificielle (écloserie, triploïde) qui se coupe de son lien avec l'environnement. Ainsi certains représentants de la profession ont su utiliser les résultats des programmes de recherche américains puis européens sur le rôle des huîtres et plus particulièrement des bancs d'huîtres comme « sentinelle » de l'environnement ${ }^{10}$. Aux États-Unis, des programmes de restauration de bancs d'huîtres sont justifiés par l'amélioration de la biodiversité, la défense des côtes et tous les « services » fournis par les coquillages dans les écosystèmes (Northern economics inc. 2009). Ces nouveaux concepts permettent de replacer l'huître dans une perspective environnementale, constitutive de la biodiversité des écosystèmes littoraux. La définition de la durabilité de l'ostréiculture est un des enjeux futurs.

Comme on le voit, le caractère aléatoire de cette profession est renforcé par la vulnérabilité du coquillage qu'elle élève, l'huître. Depuis près de 150 ans en France, la fragilité ou la disparition des huîtres (plates, portugaise, japonaise) le confirment. La triploïde et le recours aux naissains d'écloserie marquent-ils la fin du voyage de l'huître? À moins que l'ostréiculteur ne retrouve sa raison d'être: voir les écosystèmes littoraux et les huîtres-mères pourvoyeuses de naissains comme le creuset de leur métier. Aujourd'hui, ces questions restent entières. 


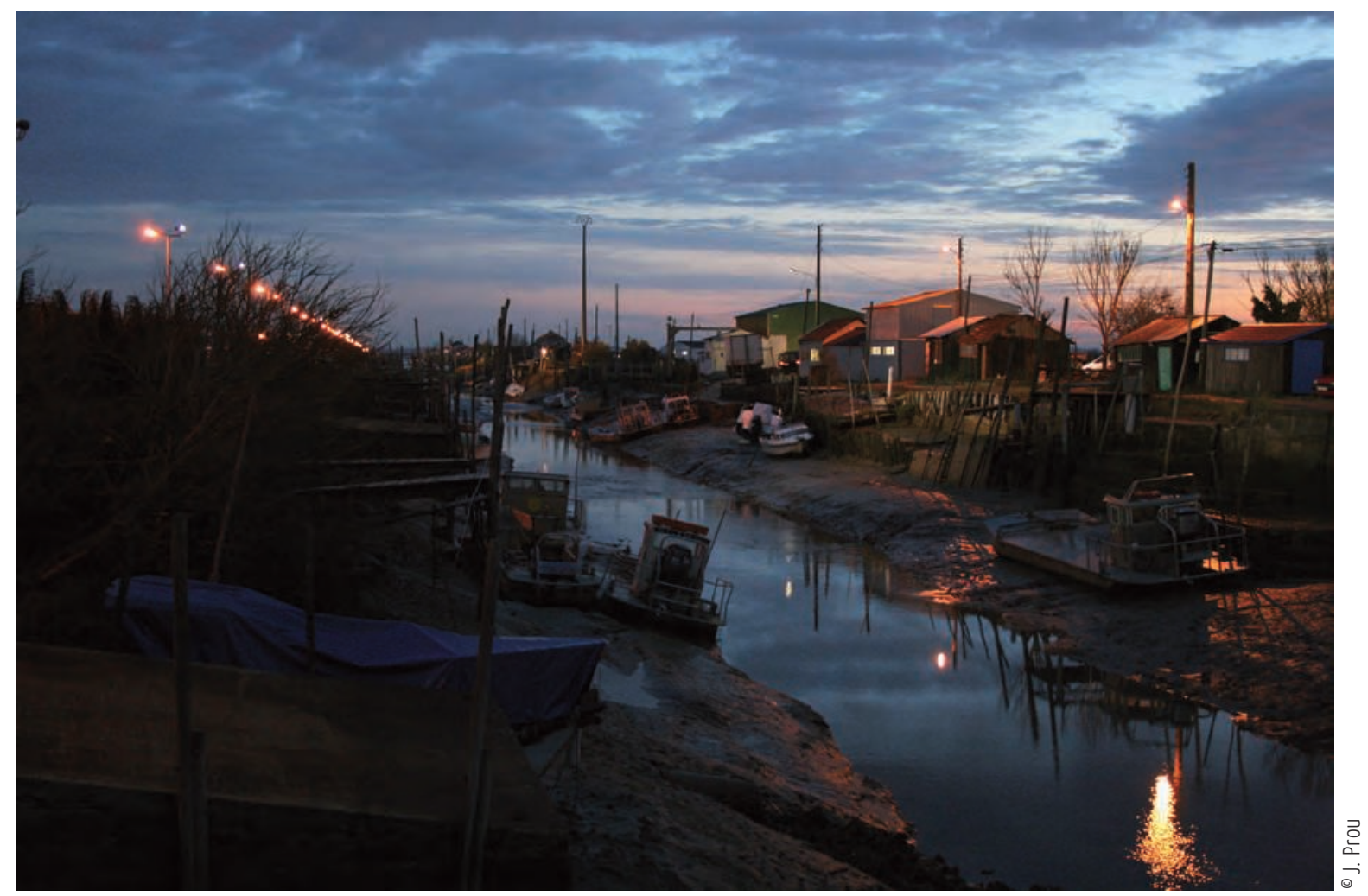

Le chenal de La Tremblade (17) regroupe, sur ses deux rives, des établissements ostréicoles. Le chenal, bordé de « cabanes » est par le jeu des marées, le lieu d'évacuation des eaux des marais et des bassins versants. 


\section{NOTES}

Photo d'ouverture: Chaland à fond plat permettant le transport de poches d'huîtres de la cabane aux parcs. Ces embarcations autrefois en bois sont maintenant en aluminium. Leur légèreté et le fond plat permet à l'ostréiculteur de se déplacer rapidement au plus près des élevages (J. Prou).

L'astérisque qui suit certains termes choisis renvoie, dans le cas de cet article, à un lexique (Cf. p. 302-303).

1. Autres initiateurs: le milieu du XIX ${ }^{e}$ siècle voit la construction des premières stations marines (Fischer, 2002) (Concarneau en 1859, Arcachon 1867, Roscoff 1872, Banyuls 1882) et l'implantation sur le littoral de chercheurs (Carbonnel, Davaine, De Quatre-fages, Lacaze-Duthiers, Bouchon-Brandely, etc.) dont les études sur la biologie des organismes vivants débouchèrent sur des applications concernant la reproduction de l'huître dans son environnement.

2. Dans le Morbihan, dès 1878 , le Conseil général vote une résolution (Anonyme, 1878) pour empêcher l'importation des huîtres portugaises et ainsi sauvegarder l'huître « armoricaine », craignant que cette huître n'envahisse ses côtes. Les bretons cultivèrent l'huître plate jusqu'à l'épisode de mortalités des années 1960 dues à deux maladies la bonamiose et la marteilose.

3. La production de naissains sélectionnés est une des raisons d'être des écloseries. La maîtrise de la fécondation permet de produire des animaux ayant des caractéristiques particulières. Chez l'huître, la triploïdisation présente, au plan commercial, l'avantage d'une croissance plus rapide puisque l'énergie passée dans la reproduction est réorientée vers la croissance. De plus, la quasi-absence de maturation pendant le printemps et l'été, permet une meilleure conservation du produit pendant ces mois et surtout offre des opportunités de consommation estivale pour des huîtres qui ne sont pas « en lait ». Parce qu'il est impossible de différentier à l'œil nu une huître diploïde d'une triploïde, la réglementation n'oblige, pour l'instant à aucun marquage particulier. Seule l'empreinte du collecteur sur la coquille permet de différencier une huître captée dans le milieu naturel d'une huître née en écloserie, qu'elle soit diploïde ou triploïde. Sur le marché français, une huître sur deux serait triploïde (Le Monde, 27 août 2009 «Les triploïdes - huîtres stériles - font des petits » : http://ribaut.blog.lemonde. fr/2009/08/27/les-triploides, consulté le 8 mars 2013).

4. Évocation de l'ouvrage de Nicolas Bouvier, L'usage du monde (1963), 364 pages, Payot (poche), 1992.
5. Suite aux mortalités, les écloseries privées se sont lancées dans des plans de sélection génétique visant à fournir à terme aux professionnels des lots d'huîtres présentant des performances de survie améliorées.

6. Commission decision 2010/221/EU of April 2010 approving national measures for limiting the impact of certain diseases in aquaculture animals and wild aquatic animals in accordance with Article 43 of Council Directive 2006/88/EC.

7. Note de service DGAL/SDSPA/N2012-8101 en date du 14 mai 2012 relative à la procédure à suivre dès le signalement d'un phénomène de mortalité massive et collective de naissains d'huîtres creuses (Crassostrea gigas) sur une zone - Mesures conservatoires de gestion des transferts des naissains d'huîtres creuses Crassostrea gigas en cas de hausse de la mortalité.

8. Les Schémas des Structures des Exploitations de Cultures Marines et les Schémas Régionaux de Développement de l'Aquaculture Marine sont soumis à évaluation environnementale.

9. Note de service DPMA/SDAEP/N2010-9618 en date du 6 juillet 2010 relative à l'exploitation des gisements naturels d'huîtres creuses (Crassostrea gigas) par les ostréiculteurs pour la récolte de naissains et/ ou juvéniles.

10. Aux États-Unis, des programmes de restaurations de bancs d'huîtres sont justifiés par l'amélioration de la biodiversité, la défense des côtes et tous les « services » fournis par les coquillages dans les écosystèmes (Northern economics inc., 2009). 


\section{LEXIQUE}

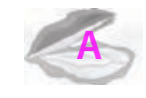

AOP: Créé en 1992, ce label protège «la dénomination d'un produit dont la production, la transformation et l'élaboration doivent avoir lieu dans une aire géographique déterminée avec un savoir-faire reconnu et constaté ».

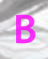

Banc, n.m. - Amas d'huîtres, de sable ou de vase, formant un dépôt et découvrant à des intervalles plus ou moins réguliers selon les coefficients de marées (Petit Robert, 2009). Nom donné à des accumulations d'huîtres vivantes. Zone de reproduction naturelle de l'huître. Les termes gisement ou crassat sont aussi employés.

Bassin naisseur: Se dit des sites conchylicoles dans lesquels le captage des naissains d'huîtres est possible. Ces sites alimentent en juvéniles les sites qui ne possèdent pas cette faculté.

Belon, n.f. - Huître plate (Ostrea edulis) nommée Belon, provenant de la rivière de Belon. Elle peut être dénommée de façon différente selon les sites de production. V. aussi Gravette et Marennes.

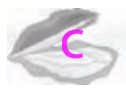

Captage, n.m. - Premiers temps d'élevage ayant pour fonction de tenter de fixer le naissain sur des collecteurs.

Collectage, n.m. - Action de collecter, ramasser (Petit Robert, 2009). Terme récemment employé pour désigner l'action de captage.

Coite, adj. - "Elle (I'huître) n'entretient aucune relation de familiarité à l'égard de l'homme. En revanche, celle de l'homme à l'égard de ce coquillage se traduit parl'emploi d'un vocabulaire anthropomorphique très évocateur qui, en outre, semble accorder à l'huître un "esprit" qu'elle n'a pas ». (Legué P., 2004 : 207)

Claire, n.f. - Bassin creusé dans les terrains argileux du littoral en vue de l'affinage des huîtres. La claire est la plus petite unité constituant le marais.

Collecteur, n. m. - Support disposé par l'ostréiculteur dans les parcs de captage en mer servant à capter les larves d'huîtres et à fixer le naissain qui pourra ensuite s'y développer. Divers matériaux sont utilisés (ardoise, bois, coquille, fer, pierre, plastique, tuile, etc.)

Coquillage, n.m. - Mollusque marin pourvu d'une coquille (Petit Robert, 2009).

Creuse, n.f. - En France, huîtres Crassostrea angulata et Crassostrea gigas qui présentent une coquille inférieure bombée.

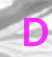

Diagénèse, n.f. - Ensemble des changements biochimiques et physicochimiques affectant un sédiment après son dépôt. Transformation progressive d'un dépôt en roche.
Écloseur,n.m. - Entrepreneur du secteur conchylicole qui produitet fournit du naissain d'huîtres obtenu par fécondation artificielle. L'entreprise est une écloserie et est souvent doublée d'une nurserie qui permet d'assurer, grâce à la production de micro-algues, les premières semaines de vie du naissain avant commercialisation aux ostréiculteurs.

Éduquer: Pourvoyager et être commercialisé, l'huîtrier commel'ostréiculteur peuvent « apprendre » aux huîtres à ne plus s'ouvrir (rester coite) pour qu'elle ne perde pas leur eau en les plongeant, puis les sortant de l'eau selon un rythme particulier. On dit aussi « tromper» les huîtres.

Élève, n.m. - 1. Jadis synonyme d'élevage: on parlait de l'élève de l'huître pour élevage de l'huître. 2. Les huîtres éparées (disséminées, semées) dans un vivier (parc à plat) étaient autrefois appelées élèves.

Estran, n. m. - Géogr. Portion du littoral entre les plus hautes et les plus basses mers (Petit Robert, 2009).

Établissement, n.m. - Bâtiment de dimensions variables servant d'abri pour les travaux ostréicoles effectués à terre.

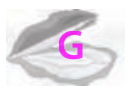

Gravette, n.f. - Autre nom de l'huître plate (Ostrea edulis) sur le Bassin d'Arcachon. V. aussi Belon et Marennes.

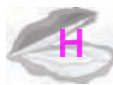

Huîtrier, n.m. - Éleveur d'huîtres (ancien).

Huîtrière, n.f. - Autre nom donné aux bancs, aux gisements d'huîtres

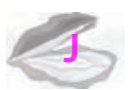

Japonaise, n.f. - Huître Crassostrea gigas élevée en France depuis les années 1970, importée à cette époque du Japon et du Canada pour remplacer la portugaise, décimée par des maladies.

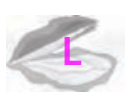

Larvaire, adj. - 1. Propre aux larves. 2. Fig. Qui n'est pas encore développé, achevé (Petit Robert, 2009).

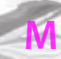

Maline, n.f. - Marée où le coefficient dépasse le degré de 70 et descend le plus, ce qui permet le travail des ostréiculteurs dans leurs parcs. "La maline des fêtes».

Marais, n.m. - Le marais définit l'espace géographique privé, qui s'oppose à l'ensemble des surfaces exploitées en mer sur le Domaine Public Maritime. 
- Un marais désigne le territoire détenu par un propriétaire.

- Un marais représente un groupe de claires alimentées par un même ruisson. Un champ de claires désigne le même espace.

Marennes, n.f. - Autre nom de l'huître plate (Ostrea edulis) en Charente inférieure (Charente-Maritime). V. aussi Belon et Gravette.

Marennes-Oléron: Marque déposée à I'Institut national de la propriété industrielle (INPI). Huîtres de Marennes-Oléron.

Marin et paysan: Statut de l'ostréiculteur.

Métamorphose, n.f. - La métamorphose est une transformation d'un animal pour passer du stade larvaire au stade adulte.

Migration, n.f. -1 . Déplacement de populations qui passent d'un pays dans un autre pours'yétablir. 4. Déplacement d'un (organisme) au cours de son développement ou de ses métamorphoses (Petit Robert, 2009).

Muscle adducteur: Chez l'huître et d'autres mollusques bivalves, les deux valves de la coquille sont reliées par un muscle qui permet leur fermeture.

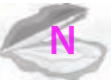

Naissain, n.m. - Ensemble des jeunes huîtres fixées sur un support naturel ou artificiel.

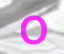

Ostréiculture, n.f. - Terme employé dès 1864 et mentionné pour la première fois dans le Littré en 1877 (tome III - ostréicole, ostréiculteur 1882). À cette époque, impuissants à maîtriser la reproduction, la seule intervention des professionnels fut de capter les larves d'huîtres. L'huître n'est donc pas, à proprement parler, à l'égal de l'agriculture, un animal cultivé.

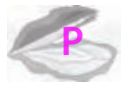

Palpes labiaux: Organes de l'huître situés autour de la bouche et ayant pour fonction l'acheminement de la nourriture.

Parcs impériaux: Zones de l'estran du bassin d'Arcachon, gérées par le service de la Marine dans les années 1860 et destinées à collecter du naissain d'huîtres plates et à repeupler les bancs naturels.

Pédivéligère, adj. ou n.f. - Stade de développement de la larve d'huître caractérisé par la présence d'un velum et d'un pied juste avant la fixation sur un support.

Plate, n.f. - Huître Ostrea edulis, historiquement présente en Europe et qui a une forme plate en comparaison de l'huître creuse.

Polyploïdisation, n.f. — La polyploïdisation est la multiplication naturelle ou artificielle du nombre des chromosomes. À l'état naturel, les cellules animales possèdent deux jeux de chromosomes (diploïdes), les animaux triploïdes en possèdent, trois et les tétraploïdes, quatre. Les polyploïdes à nombre impair de chromosomes sont réputés stériles, c'est-à-dire que, même s'ils peuvent présenter une maturation des cellules sexuelles, ces cellules ne donnent pas lieu à fécondation viable. La production d'huître triploïde s'effectue dans les écloseries.

Portugaise, n.f. - Huître Crassostrea angulata, importée à partir de 1866 de l'estuaire du Tage et qui a été élevée en France jusqu'à sa disparition dans les années 1970.

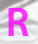

Rogue, n.f. — Eufs de morue ou de hareng utilisés comme appât pour la pêche à la sardine (Petit Robert, 2009). Aujourd'hui plus généralement employé pour désigner un appât constitué des œufs de certains poissons.

Roulante, n.f. - Huître détachée de son support, apportées par la mer sur le bord de plage, provenant de parc ou de gisements.

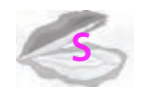

Sessile, adj. — Qualifie la faune aquatique qui vit fixée au sol.

Sentinelle (de l'environnement): La capacité de filtration de l'eau de mer confère à l'huître une fonction de reflet de la qualité de l'environnement. Les réseaux de surveillance de la qualité du milieu marin utilisent les huîtres et les moules comme indicateurs des pollutions issues des bassins versants.

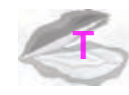

Transhumance, n.f. - Migration périodique du bétail de la plaine, qui change de pacage en été et s'établit en montagne (Petit Robert, 2009).

Trompage, n.m. - Dernière opération avant l'expédition des huîtres consistant à leur apprendre à rester fermées lorsqu'elles sont à sec de manière à conserver un maximum de liquide à l'intérieur des coquilles et à parvenir vivantes aux consommateurs.

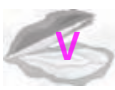

Vagile, adj. - Qualifie un organisme capable de se déplacer dans son environnement.

Véligère, adj. ou n.f. — Stade de développement de la larve d'huître caractérisé par la présence d'un velum.

Velum, n.m. - Sorte de voile cilié, que la larve d'huître déploie autour de sa coquille lorsqu'elle nage et qui lui sert à capter les algues microscopiques constituant sa nourriture. 


\section{RÉFÉRENCES}

Anonyme 1878 Parcs à huîtres - Huîtres de Portugal. Rapports du Préfet et délibérations du Conseil général. Conseil général du Morbihan 8: 175.

1714 Ordonnance de la marine, du mois d'aoust 1681 ([Reprod.])/[France]. Paris: C. Osmont.

Barbier, A. 1886 Un parc à huîtres à Poitiers. Bulletins de la Société des antiquaires de l'Ouest 2(4) : 468-473

Barbosa du Bocage, J. V. 1868 Considerações ácerca do melhor aproveitamento das ostreiras da margem esquerda do Tejo e da cultura das nossas ostras. Lisboa: Typographia da Academia.

Barnabé, G. (dir.) [1986] 1989 (2éd.) Aquaculture. Paris: Lavoisier, (Tec \& Doc) 2 vol. XXV-VII, 1308 p.

Barrau, J. 1985 Écosystème, civilisations et sociétés humaines: le point de vue du naturaliste, Information sur les sciences sociales, t. XIV (1).

Bouchon-Brandely 1882 On the Sexuality of the Common Oyster (O. edulis), and that of the Portuguese Oyster (O. angulata). Artificial Fecundation of the Portuguese Oyster. Bulletin of the United States Fish Commission: 339-341.

Bouvier, N. 1992 [1963] L’usage du monde. Payot (poche).

Callon, M., 1986 Élément pour une sociologie de la traduction: la domestication des coquilles Saint-Jacques et des marins pêcheurs dans la baie de Saint-Brieuc. L’année sociologique numéro spécial: La sociologie des sciences et techniques (36): 169-208.

Champouillon, 1876 Sur les propriétés des huîtres portugaises. Comptes rendus hebdomadaires des séances de l'Académie des sciences 82: 1111-1112.

Coste, V. 1861 Voyage d'exploration sur le littoral de la France et de l'Italie. 2e éd. Paris: Imprimerie Impériale.

Chevalier, L., 1983 Les relais de mer. Paris: Fayard, 455 p.

Diderot, D. 1751-1765 Encyclopédie ou Dictionnaire raisonné des sciences, des arts et des métiers (17 vol.). Paris: Briasson, David, Le Breton, Durand; Neuchâtel: Faulche S. et Cie.

Diederich, S. et al. 2005 Introduced Pacific Oysters (Crassostrea gigas) in the Northern Wadden Sea: Invasion Accelerated by Warm Summers? Helgoland Marine Research 59(2) : 97-106.

Digard, J.-P. 1990 L'homme et les animaux domestiques. Anthropologie d'une passion. Paris: Fayard.

Fischer, P. 1880 Sur les conditions d'existence de l'Ostrea angulata, Lamarck. Journal de conchyliologie 3(20) : 83-86.

Fischer, J.-L. 2002 Créations et fonctions des stations maritimes françaises. La revue pour l'histoire du CNRS 7, mis en ligne le 17 octobre 2006, consulté le 21 mai 2012. URL: http://histoire-cnrs.revues.org/537

Grelon, M. 1978 Saintonge, pays des huitres vertes. La Rochelle: Rupella.

Gutiérrez-Zugasti, I., et al. 2011 Shell Midden Research in Atlantic Europe: State of the Art, Research Problems and Perspectives for the Future. Quaternary International 239 (1-2): 70-85. Web. 24 May 2012.

Hervé, P. 1935 Les huîtres : les bancs d'huitres naturels, les portugaises, les huîtres dans l'île d'Oléron, les huîtres à Marennes [Texte imprimé], Marennes: A. Barbault.

Lapègue, S., et al. 2004 Evidence for the Presence of the Portuguese Oyster, Crassostrea angulata, in Northern China. Journal of Shellfish Research 23(3) : 759-763.

Legué Dupont P. 2004 La moisson des marins-paysans. L'huître et ses éleveurs dans le bassin de Marennes-Oléron. Paris: Éditions MSH-INRA.

Legué, P. 2000 L'évolution du foncier ostréicole dans le bassin de Marennes-Oléron. Études foncières 88: 29-32.

Legué-Dupont P. 1987 Ethnologie des entreprises ostréicoles du bassin de Marennes-Oléron. http://archimer. ifremer.fr/doc/00014/12495

Leroux $D^{\mathrm{r}}$., 1882 Présentation de produits d'ostréiculture pouvant faire croire à l'hybridation. Comptes-rendus de la $11^{e}$ session La Rochelle: Association française pour l'avancement des sciences: 474-475.

Northern Economics, Inc. 2009 Valuation of Ecosystem Services from Shellfish Restoration, Enhancement and Management: A Review of the Literature. Washington: Pacific Shellfish Institute.

Papy, L. 1941 La côte atlantique de la Loire à la Gironde. Bordeaux: Delmas, 302 p.

Pelosse, V., Micoud, A. 1993 Du domestique au sauvage cultivé: des catégories pertinentes de la biodiversité? Études Rurales 129-130: 9-14. 
Ponge, F. 1989 [1942] Le parti pris des choses. Paris: Gallimard (Poésie), 224 p.

Schmidt, A., Wehrmann, A. et Dittmann, S. 2008 Population Dynamics of theInvasive Pacific Oyster (Crassostrea gigas) During the Early Stages of an Outbreak in the Wadden Sea (Germany). Helgoland Marine Research 62(4): 367-376.

Steele, E. N. 1964 The Immigrant Oyster (Ostrea gigas) Now Known As the Pacific Oyster. Washington: Olympia / Warren's Quick Print. In cooperation with Pacific Coast Oyster Growers Association, Inc.

Tchernia, A. 1997 Le cercle de L. Licinius Crassus et la naissance de la hiérarchie des vins à Rome. Comptes-rendus des séances de l'Académie des Inscriptions et Belles-Lettres 141(4) : 1247-1259.

White, J., Ruesink, J. L, et Trimble, A. C. 2009 The Nearly Forgotten Oyster: Ostrea lurida Carpenter 1864 (Olympia oyster) History and Management in Washington State. Journal of Shellfish Research 28(1) : 43-49.

Wrange, A.-L., et al. 2010 Massive Settlements of the Pacific Oyster, Crassostrea gigas, in Scandinavia. Biological Invasions 12(5) : 1145-1152

\section{RÉSUMÉ}

L'huître, un coquillage nomade sans tête ni jambe mais avec un pied. L'huître sauvage est un animal qui après deux ou trois semaines de vie au gré des courants marins, se fixe sur un support et passe le reste de sa vie, enfermé dans une coquille hermétique qui la protège. Source d'alimentation pour la subsistance et source de plaisir, l'homme a fait de l'huître vouée à l'immobilisme, une grande voyageuse pour la consommer crue et vivante en des lieux très éloignés de son lieu originel de vie. L'histoire de l'ostréiculture illustre les voyages de cet animal dont les différentes espèces se succèdent pour le plaisir des hommes. La destinée de l'huître, produit vivant, influe fortement les groupes sociaux qui l'exploitent. La disparition d'une espèce et l'adoption d'une nouvelle, comme cela fut le cas pour l'huître plate et l'huître portugaise, induisent des bouleversements techniques, culturels et scientifiques. Récemment, les avancées de la recherche lui offrent encore de nouvelles possibilités de voyage mais non sans risque...

\section{ABSTRACT}

The Oyster, a nomad shellfish with no head nor leg but a foot. After spending a couple of weeks carried away by ocean currents, wild oysters larvae settle themselves on a hard surface and get ready to spend a lifetime quietly trapped inside a shell that is also their shelter. Using oysters as a source of food and pleasure, men have changed their fate. Far from remaining immobile, often transported faraway from their home places, they have become world travellers. The destiny of oysters, a peculiar live in-shell product, has great influence on that of social groups that exploit them. The decline of a species and its replacement by another one, as was the case of the flat oyster superseded by the Portuguese oyster, have the potential to lead to major changes at technical, cultural and scientific levels. The history of shellfish farming is an illustration of the expeditions undertaken by this animal, which various species make man's delight. Current research progress has provided brand new opportunities for oyster to continue travelling but the journey is not risk free...

\section{MOTS CLÉS}

Huîtres, ethnologie, biologie, voyages, terre-mer, ostréiculture, triploïde

\section{KEYWORDS}

Oysters, anthropology, biology, journeys, land-sea, triploid 